\title{
Orientação Profissional no ensino odontológico brasileiro
}

\author{
Suzely Adas Saliba Moimaz*, Adrielle Mendes de Paula Gomes**, Tânia Adas Saliba***, Artênio José \\ Isper Garbin****, Cléa Adas Saliba Garbin*
}
* Professora Titular, Departamento de Odontologia Infantil e Social, Faculdade de Odontologia de Araçatuba/UNESP
** Doutora em Odontologia Preventiva e Social, Departamento de Odontologia Infantil e Social, Faculdade de Odontologia de Araçatuba/UNESP
*** Professora Adjunta, Departamento de Odontologia Infantil e Social, Faculdade de Odontologia de Araçatuba/UNESP
**** Professor Adjunto, Departamento de Odontologia Infantil e Social, Faculdade de Odontologia de Araçatuba/UNESP

Recebido em 17/07/2018. Aprovado em 30/09/2019.

\begin{abstract}
RESUMO
Neste estudo objetivou-se analisar os conteúdos de "Orientação Profissional" nas estruturas curriculares dos cursos de graduação em Odontologia do Brasil. As variáveis estudadas foram nomenclatura, período ministrado, carga horária e características das aulas (teórico/prático). Foi também realizada análise qualitativa dos conteúdos descritos nos planos de ensino das disciplinas. No endereço eletrônico do Conselho Federal de Odontologia foi obtida a lista com os 242 cursos de Odontologia. Destes, 192 disponibilizavam o plano de ensino, projeto pedagógico ou matriz curricular. Do total, 72,2\% apresentavam alguma disciplina relacionada à "Orientação Profissional" e 3,2\% eram ofertadas como disciplinas optativas. A carga horária média era de 46,5 $\pm 26,96$ horas (mediana $=40$ ), sendo ministrada com maior frequência entre o $3^{\circ}$ e $4^{\circ}$ semestres $(33,9 \%)$. Na maioria dos cursos $(54,4 \%)$ os conteúdos são trabalhados de forma teórica. A nomenclatura apresenta variações, sendo a mais utilizada "Orientação Profissional". Conclui-se que em todas variáveis estudas há uma grande heterogeneidade, destacando-se, assim, a dificuldade do ensino do tema de forma articulada e contínua nos cursos.
\end{abstract}

Descritores: Faculdades de Odontologia. Educação Superior. Ensino.

\section{INTRODUÇÃO}

No ensino odontológico brasileiro, a formação de um profissional com visão generalista, humanista, crítica e reflexiva foi preconizada pelas
Diretrizes Curriculares Nacionais $(\mathrm{DCN})^{1}$. Ao contrário do currículo mínimo anteriormente adotado, que detalhava excessivamente os conteúdos obrigatórios, inibindo a inovação e a 
criatividade, as DNC oportunizaram a elaboração de projetos pedagógicos de acordo com a realidade local das instituições formadoras ${ }^{2}$.

Existe um forte apoio para que haja mudança nos currículos das escolas de Odontologia, principalmente voltada à educação baseada em competências ${ }^{3}$. Porém, a organização curricular na maioria das instituições brasileiras é tradicional, com conteúdos fragmentados e estanques, onde seus teores são integrados somente na última etapa de formação do cirurgião-dentista, o que torna complexa tal transformação nos modelos de ensino $^{4-7}$.

Em menor número de instituições, a organização curricular é estabelecida em módulos integrados, por complexidade, baseados em problemas extraídos da realidade ${ }^{6}$, onde se espera a formação de profissionais mais humanizados, competentes e preocupados em cuidar do ser humano e não apenas da doença ${ }^{8}$. Essa metodologia sugere a quebra do modelo tradicional, estimulando o aluno à autoaprendizagem, aprender a aprender e busca autônoma do conhecimento ${ }^{9}$.

Formar um cirurgião-dentista integrado à realidade profissional é um desafio a ser conquistado durante a graduação e que deve transcender o limite da Universidade. Essa transição da Universidade para o mercado de trabalho, por vezes, é considerada angustiante para o recém-formado.

Em geral, as disciplinas do curso de Odontologia são marcadas pela prática individual, voltadas para o aprendizado de técnicas e materiais, restringindo-se ao campo operacional, fazendo com que os estudantes se concentram fortemente nos procedimentos dentários ${ }^{10}$. Há necessidade de se trabalhar o aprendizado do aluno sobre componentes curriculares importantes estabelecidos nas DCN, que vão proporcionar ao mesmo capacidade de tomada de decisão, de liderança, de inserção do mercado de trabalho, de conhecimento das reais necessidades da população $^{11}$.

Nesse sentido, com o intuito de auxiliar na formação do futuro cirurgião-dentista, a disciplina de "Orientação Profissional" torna-se imprescindível nos cursos de graduação, podendo ser considerada uma base para a vida profissional futura, um conjunto de normas e técnicas capaz de preparar os acadêmicos para enfrentar as incoerências mercadológicas e para um eficiente exercício da profissão ${ }^{12}$. Em interface com a Saúde Coletiva, Odontologia Legal e Bioética, a disciplina pode assumir diferentes nomenclaturas, de acordo com o Projeto Pedagógico de cada Instituição de Ensino Superior (IES).

Devido à carência de estudos e artigos publicados sobre o tema, essa pesquisa tem por objetivo analisar os conteúdos de "Orientação Profissional" nas estruturas curriculares dos cursos de graduação em Odontologia do Brasil.

\section{METODOLOGIA}

Trata-se de um estudo descritivo e de análise documental sobre as disciplinas que contemplam a "Orientação Profissional" no curso de Odontologia nas instituições de ensino superior do Brasil.

No endereço eletrônico do Conselho Federal de Odontologia ${ }^{13}$, foi obtida a lista com 242 cursos e seus respectivos endereços eletrônicos. Desse total, três estavam com o curso suspenso ou extinto, restando 239 instituições em atividade regular.

Foram realizadas consultas nos endereços eletrônicos dessas instituições, disponíveis para acesso público e obtidas informações sobre plano de ensino, projeto pedagógico ou matriz curricular de 192 cursos. Foram estabelecidos como critério de exclusão as instituições cujos endereços eletrônicos não estavam disponíveis (n=9) e as que não disponibilizaram informações sobre as disciplinas oferecidas $(\mathrm{n}=38)$.

Foi realizada análise de conteúdo, conforme Bardin (1988) ${ }^{14}$, das ementas das disciplinas que 
tinham em sua nomenclatura "Orientação Profissional", a fim de verificar quais assuntos eram mais abordados. A categorização final foi feita por meio do reagrupamento dos conteúdos.

A partir desta análise, realizou-se busca por palavras-chave para verificar a existência de disciplinas correlatas e analisar as variações de nomenclaturas. Em seguida, as disciplinas correlatas também passaram pela análise de conteúdo das ementas, a fim de quantificar os assuntos abordados pelas mesmas.

Após leitura criteriosa de todos os documentos, estudaram-se as seguintes variáveis: natureza administrativa das instituições, semestre em que a disciplina é ministrada, carga horária total e característica das aulas (teóricas e/ou prática).

Os dados obtidos foram digitados $\mathrm{e}$ submetidos à análise quantitativa descritiva e os resultados foram explanados em forma de quadros e figuras.

\section{RESULTADOS}

Do total de cursos de Odontologia analisados $(n=192), 27,6 \% \quad(n=53)$ pertencem a instituições públicas e $72,4 \%(n=139)$ privadas.

A tabela 1 apresenta a análise de conteúdo feita a partir das ementas disponíveis das disciplinas de "Orientação Profissional" de 25 cursos de Odontologia, com o objetivo de verificar a frequência dos assuntos mais abordados.

Tabela 1. Análise de conteúdo das disciplinas de "Orientação Profissional”, a partir das ementas disponíveis

\begin{tabular}{lcc}
\hline \multicolumn{1}{c}{ Assuntos abordados } & n & \% \\
\hline Administração (Marketing/Propaganda/Empreendedorismo/Gestão) & 17 & 34,0 \\
Ergonomia & 16 & 32,0 \\
Biossegurança & 7 & 14,0 \\
Odontologia Legal e Bioética & 5 & 10,0 \\
Psicologia & 2 & 4,00 \\
Saúde Coletiva & 1 & 2,0 \\
Metodologia da Pesquisa & 1 & 2,0 \\
Bioestatística & 1 & 2,0 \\
\hline
\end{tabular}

A partir da análise de conteúdo, para verificar a existência de disciplinas correlatas e analisar as variações de nomenclaturas, foi feita uma nova busca nas grades curriculares, pelas seguintes palavras-chave: Administração (Marketing, Propaganda, Empreendedorismo, Gestão), Biossegurança e Ergonomia.

As nomenclaturas de disciplinas relacionadas à "Orientação Profissional" encontradas neste estudo foram relacionadas na tabela 2. Dentre elas, 72,9\% ( $\mathrm{n}=140)$ apresentavam em sua matriz alguma disciplina que referenciasse a qualquer conteúdo de "Orientação Profissional"; destas, 3,2\% ( $n=6)$ eram ofertadas como disciplinas optativas.
Uma nova análise de conteúdo foi feita, a partir das ementas disponíveis, para verificar os conteúdos mais abordados pelas disciplinas "Orientação Profissional" e disciplinas correlatas. O conteúdo relacionado à "Ergonomia" estava presente em $31,3 \%$ das ementas, seguida por "Biossegurança" (30,3\%) e "Administração" $(19,2 \%)$. Outros assuntos encontrados foram: Odontologia Legal e Bioética (12,1\%), Psicologia (3,0\%), Saúde Coletiva (2,0\%) e Metodologia da Pesquisa (2,0\%).

A carga horária média dos cursos de Odontologia analisados foi de 4.449 horas (mínimo de 4.000 h e máximo de 5.595h). Na análise da carga horária das disciplinas, observou-se uma 
média de 46,5 $\pm 26,96$ horas (mediana $=40$ ).

O mínimo observado foi de 15 horas e o máximo de 300 horas. De acordo com a figura 1, a maioria dos cursos $(64,0 \%)$ dedica de 15 a 44 horas à disciplina.

Com relação ao momento em que as disciplinas eram ministradas, a maioria das faculdades $(33,9 \%)$ as disponibilizavam no $3^{\circ}$ ou $4^{\circ}$ semestre do curso (figura 2). De forma geral, a disciplina pode ser observada em todas as etapas do curso, com exceção do último ano $\left(11^{\circ}\right.$ e $12^{\circ}$ semestres).

Tabela 2. Distribuição percentual das nomenclaturas das disciplinas relacionadas à Orientação Profissional nos cursos de Odontologia brasileiros

\begin{tabular}{|c|c|}
\hline Nomenclatura & $\%$ \\
\hline $\begin{array}{l}\text { Orientação Profissional/ Orientação Profissional em Odontologia/ Orientação Profissional } \\
\text { Odontológica }\end{array}$ & 26,1 \\
\hline $\begin{array}{l}\text { Biossegurança/ Biossegurança aplicada à Odontologia/ Biossegurança em Odontologia/ } \\
\text { Biossegurança e Saúde Ambiental/ Biossegurança e Orientação Profissional Odontológica/ } \\
\text { Educação Ambiental e Biossegurança }\end{array}$ & 14,1 \\
\hline $\begin{array}{l}\text { Biossegurança e Ergonomia/ Ergonomia e Biossegurança/ Biossegurança e Ergonomia aplicada à } \\
\text { Odontologia/ Biossegurança e Ergonomia em Odontologia/ Princípios Básicos de Ergonomia e } \\
\text { Biossegurança/ Biosseguraça e Ergonomia, Saúde do trabalhador e doenças ocupacionais/ } \\
\text { Biossegurança, Ergonomia e Saúde do Trabalhador }\end{array}$ & 13,2 \\
\hline $\begin{array}{l}\text { Gestão e Planejamento em Odontologia/ Planejamento e Gestão para o Mercado/ Gestão em Saúde } \\
\text { e Marketing/ Gestão empreendedora/ Gestão Empresarial/ Gestão Odontológica/ Gestão } \\
\text { Profissional/ Gestão de Saúde: Odontologia/ Gestão e Marketing Aplicado à Odontologia/ Gestão e } \\
\text { Marketing em Odontologia/ Gestão e Marketing Odontológico/ Gestão de Serviços Odontológicos/ } \\
\text { Gestão o o Mundo do Trabalho/ Liderança e Empreendedorismo/ Marketing e Empreendedorismo/ } \\
\text { Marketing e Gestão Empresarial/ Marketing e Publicidade/ Marketing em Odontologia/ Marketing } \\
\text { para Profissionais Liberais/ Implantação e Gestão de Clínica Odontológica/ Gerenciamento da } \\
\text { Atividade Odontológica/ Gestão aplicada à Odontologia e Orientação Profissional }\end{array}$ & 13,2 \\
\hline $\begin{array}{l}\text { Ergonomia/ Ergonomia Aplicada à Odontologia/ Ergonomia Odontológica/ Ergonomia e } \\
\text { Odontologia do Trabalho }\end{array}$ & 7,3 \\
\hline $\begin{array}{l}\text { Empreendedorismo/ Empreendedorismo Aplicado à Saúde/ Empreendedorismo e Gestão/ } \\
\text { Empreendedorismo e Marketing em Odontologia/ Empreendedorismo e Plano de Negócios em } \\
\text { Saúde/ Economia - Administração e Marketing em Odontologia/ Administração e Gerenciamento }\end{array}$ & 6,4 \\
\hline $\begin{array}{l}\text { Odontologia Legal, Bioética E Gestão Em Odontologia/ Bioética e Orientação Profissional/ } \\
\text { Deontologia, Odontologia Legal e Orientação Profissional/ Odontologia Legal, Deontologia e } \\
\text { Gerenciamento Profissional/ Odontologia Legal, Deontologia e Orientação Profissional/Legislação, } \\
\text { Deontologia e Orientação Profissional }\end{array}$ & 4,3 \\
\hline & 2,6 \\
\hline $\begin{array}{l}\text { a/ Orientação } \\
\text { lho }\end{array}$ & 2,6 \\
\hline & 0,9 \\
\hline & 0,9 \\
\hline $\begin{array}{l}\text { Outras: Agentes Infecciosos - Imunologia e Biossegurança/ Anestesiologia-Ergonomia- } \\
\text { Biossegurança/ Biossegurança, Bioética, Meio Ambiente e Emergência na Ârea de Saúde/ } \\
\text { Biossegurança e Fundamentos de Enfermagem/ Desenvolvimento Pessoal e Empregabilidade/ } \\
\text { Empreendedorismo e Sustentabilidade/ Ergonomia e Odontologia Legal/ Estágio Supervisionado 3, } \\
\text { 7, 8/ Odontologia do Trabalho/ Odontologia Preventiva e Social IV (Orientação Profissional e } \\
\text { Odontologia Legal)/ Organização do Processo de Trabalho em Saúde II/Políticas e Gestão de Saúde/ } \\
\text { Pré-Clínica I/ Projeto aplicado: Controle de infecção e biossegurança/ Riscos ocupacionais do }\end{array}$ & 8,5 \\
\hline & \\
\hline
\end{tabular}


Figura 1. Distribuição percentual dos cursos de Odontologia quanto à carga horária, em horas, destinada às disciplinas da Orientação Profissional

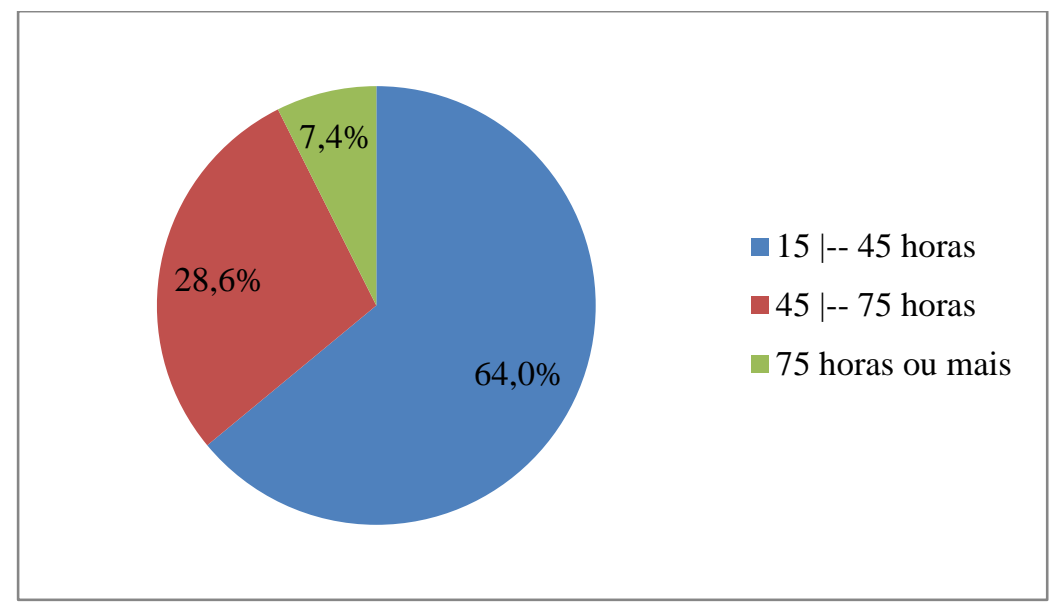

Figura 2. Distribuição percentual dos cursos de Odontologia quanto ao semestre destinados às disciplinas da Orientação Profissional

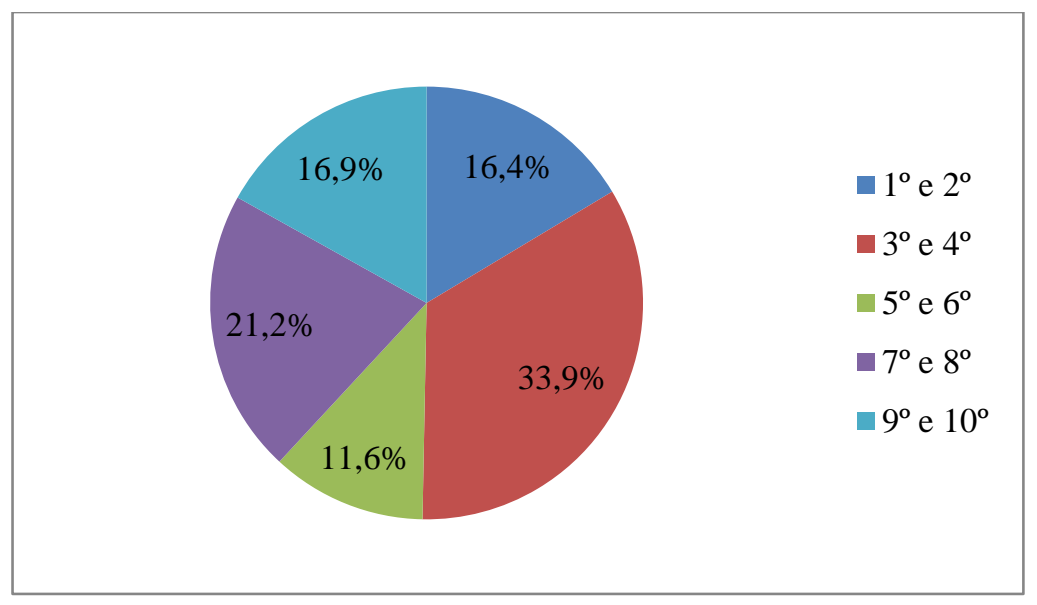

A maioria dos cursos $(60,5 \%)$ não duração do curso de Odontologia. As matérias mencionou em seu plano de ensino o caráter teórico eram divididas em Matérias Básicas (Ciências ou prático da disciplina. Dentre as que Morfológicas; Ciências Fisiológicas; Ciências mencionaram, 54,4\% ministram a disciplina Patológicas; Ciências Sociais) e Matérias somente com conteúdos teóricos e 45,6\% Profissionalizantes (Propedêutica Clínica; Clínica ministram conteúdos teóricos e práticos. Odontológica; Clínica Odontopediátrica;

\section{DISCUSSÃO}

Odontologia Social; Clínica Integrada). A

Historicamente, em 1982, o Conselho Federal de Educação, por meio da Resolução CFE $n^{\circ}$ 04/82, fixou os mínimos de conteúdo e de disciplina de Odontologia Social englobava os conteúdos sobre os aspectos sociais, deontológicos, legais e os de orientação profissional.

Essa estrutura vigorou até 2002, quando 
foram implantadas as novas diretrizes curriculares nacionais $(\mathrm{DCN})^{1}$ para os cursos de Odontologia e que propõem conteúdos essenciais, como Ciências Biológicas e da Saúde; Ciências Humanas e Sociais; e Ciências Odontológicas. Mais uma vez, não fica claro como os conteúdos de Orientação Profissional devem ser abrangidos.

Com elevado número de profissionais de Odontologia sendo formados por ano no Brasil, a responsabilidade em desenvolver habilidades e competências para diferenciar-se no mercado de trabalho, e para que os mesmos sejam capazes de atender a demanda, pode afligir os graduandos. Adequar o perfil profissional não se faz necessária unicamente para atender aos requisitos das diretrizes ${ }^{15}$.

A disciplina de "Orientação Profissional" pode subsidiar os futuros cirurgiões-dentistas para tais situações ${ }^{9}$. Porém, a formação humana e empreendedora ainda não é prioridade na maioria dos cursos de Odontologia ${ }^{16}$. O foco do ensino sempre esteve mais voltado para o caráter curativo das doenças, baseado na mais recente tecnologia do mercado ${ }^{17}$. Corroborando com estas informações, neste estudo pode-se observar que muitas Faculdades analisadas não ministram a disciplina de "Orientação Profissional", nem os conteúdos correlatos, e em 3,2\% oferecem como disciplinas optativas.

A diversidade em relação às nomenclaturas também pode ser observada, evidenciada a inexistência de consenso entre as instituições. Tais diferenças podem ser explicadas pelo fato da disciplina abordar diversos conteúdos e, assim, desassociar-se da "Orientação Profissional" e assumir-se como conteúdo isolado.

Em 2007, através da Resolução $n^{\circ}$ 2, o Conselho Nacional de Educação ${ }^{18}$ instituiu a carga horária mínima para os cursos de graduação, bacharelados, na modalidade presencial. A duração para o curso de Graduação em Odontologia foi estabelecida em 4000 horas. Neste estudo, a média da carga horária das disciplinas de "Orientação Profissional" (46,5 horas) atingiu 1,16\% da carga horária mínima do total do curso exigida pela Resolução. Visando a formação de um profissional apto a ser empreendedor, gestor, empregador ou líder na equipe de saúde, contemplado nas DCN, a carga horária da disciplina deveria ser em torno de $25 \%$ para que haja uma correta assimilação dos conteúdos pelos graduandos ${ }^{16}$.

Prevista pelas $\mathrm{DCN}^{1}$, a divisão dos conteúdos por disciplina é uma configuração utilizada pela maioria dos cursos. Porém, para atender ao pressuposto de um ensino integrado, teoria e prática, bem como disciplinas básicas e profissionalizantes não devem ser dissociadas. Evidenciou-se neste estudo, que a maioria das instituições ministra a disciplina de "Orientação Profissional" de forma isolada e somente com conteúdos teóricos. Esse tipo de estratégia de ensino não permite que ocorra aprendizagem de forma expressiva e, assim, não é capaz de atingir os objetivos de desenvolvimento de competências por parte do graduando ${ }^{6}$.

Ainda, podemos observar a variação de semestres em que a disciplina é ministrada. $\mathrm{O}$ desenvolvimento contínuo ao longo de todo o curso pode ser observado em somente em uma instituição de ensino. Isso demonstra que há uma segmentação e desarticulação entre as demais disciplinas, tornando um grande desafio a ser superado.

Partindo da ideia de que o acadêmico de Odontologia, durante o seu aprendizado, dedica uma parte significativa de seu tempo ao aprimoramento de habilidades técnicas ${ }^{19-20}$ e de que a integração dos conteúdos só ocorre nos últimos anos na maioria das instituições ${ }^{6}$, faz-se necessário que a "Orientação Profissional” esteja presente de forma integrada e contínua durante todo o curso.

Destaca-se que o grande desafio da disciplina de Orientação Profissional é de estar presente de forma articulada e contínua nos cursos de 
Odontologia do Brasil.

\section{CONCLUSÃO}

Todas variáveis estudas referentes à disciplina de "Orientação Profissional" nos cursos de Odontologia no Brasil apresentam heterogeneidade. A carga horária variou entre 15 e 300 horas, sendo os conteúdos ministrados com mais frequência no $3^{\circ}$ e $4^{\circ}$ semestres. As nomenclaturas mais utilizadas foram "Orientação Profissional", "Biossegurança", “Gestão e Planejamento" e "Ergonomia".

\section{ABSTRACT \\ Professional Guidance in Brazilian dental education}

This study aimed to analyze the contents of "Professional Guidance" in the curricular structures of undergraduate dentistry courses in Brazil. The variables studied were: nomenclature, period taught, workload and class characteristics (theoretical/practical). A qualitative analysis of the contents described in the subjects' teaching plans, was also performed. The e-mail address of the Federal Council of Dentistry obtained the list with the 242 courses of Dentistry. Of these, 192 provided the teaching plan, pedagogical project or curriculum matrix. Of the total, $72.2 \%$ had some subject related to "Vocational Guidance" and 3.2\% were offered as optional subjects. The average workload was $46.5 \pm 26.96$ hours (median $=40$ ), being given more frequently between the $3 \mathrm{rd}$ and 4th semesters (33.9\%). In most courses (54.4\%) the contents are worked theoretically. The nomenclature presents variations, being the most used "Professional Orientation". It is concluded that in all variables studied there is a great heterogeneity, thus highlighting the difficulty of teaching the theme in an articulated and continuous way in the courses.

Descriptors: Schools, Dental. Education, Higher. Teaching.

\section{REFERÊNCIAS}

1. Brasil. Conselho Nacional de Educação.
Câmara de Educação Superior. Resolução CNE/CES 3, de 19 de fevereiro de 2002. Institui Diretrizes Curriculares Nacionais do Curso de Graduação em Odontologia. Diário Oficial União, Brasília, seção1:10, 04 mar 2002. [Acesso em: 03/10/2016] Disponível em: http://portal.mec.gov.br/cne/arquivos/ pdf/CES032002.pdf

2. Toassi RFC, Souza JM, Baumgarten A, Rosing CK. Avaliação curricular na educação superior em odontologia: discutindo as mudanças curriculares na formação em saúde no Brasil. Rev ABENO. 2012;12(2):170-7.

3. Ditmyer MM, Mobley CC, Davenport WD. Evaluation of an integrative model for professional development and research in a Dental Curriculum. J Dent Educ. 2014;78(3):368-79.

4. Rodrigues MM, Reis SMAS. A interdisciplinaridade e a integração no ensino odontológico: reflexos sobre o perfil profissional em relação às reais demandas da maioria da população por atenção odontológica. Em Extensão. 2004;4(1):207.

5. Xavier LN, Oliveira GL, Gomes AA, Machado MFAS, Eloia SMC. Analisando as metodologias ativas na formação dos profissionais de saúde: uma revisão integrativa. Sanare. 2014;13(1):76-83.

6. Garcia LSG, Lopes JL, Silva BS, Tanaka EE. Organização didático pedagógica estratégias para o desenvolvimento de competências. Rev ABENO. 2016;16(2):5461.

7. Silveira JLGC, Garcia VL. Mudança curricular em Odontologia: significados a partir dos sujeitos da aprendizagem. Interface. 2015;19(52):145-58.

8. Saliba NA, Moimaz SAS, Chiaratto RA, Tiano AVP. A utilização da metodologia 
PBL em Odontologia: descortinando novas possibilidades ao processo ensinoaprendizagem. Rev Odonto Ciênc. 2008;23(4):392-6.

9. Lemos SG, Morita MC, Carloto EET. Recursos utilizados por graduandos de odontologia e Cirurgiões-Dentistas do SUS para estudar e resolver dúvidas surgidas durante $o$ trabalho. Rev ABENO. 2014;14(1):3-16.

10. Presoto CD, Wajngarten D, Garcia PPNS. Risk Factors of Musculoskeletal Disorders in Dental Students - A Qualitative Study. Br J Med Med Res. 2016;18(10):1-9.

11. Haddad AE, Brenelli SL, Passarella TM, Ribeiro TCV. Política Nacional de Educação na Saúde. Rev Baiana Saúde Pública. 2008;32(1):98-114.

12. Sales-Peres A, Silva RHA, Oliveira AN, Lima JP, Foelkel TP. Panorama da Disciplina de Orientação Profissional no Estado de São Paulo e sua importância no ensino de Fonoaudiologia. Rev Inst Ciênc Saúde. 2008;27(2):144-7.

13. Brasil. Conselho Federal de Odontologia. Lista de Faculdades de Odontologia por Estado. [Acesso em: 18/04/2016] Disponível em: http://cfo.org.br/servicos-e-consultas/ faculdades/

14. Bardin L. Análise de conteúdo. Lisboa: Edições 70; 2009.

15. Fernandes DC, Freitas DA, Pedrosa AK, Silva EM. Currículo de Odontologia e as Diretrizes Curriculares Nacionais. Revist. Port.: Saúde e Sociedade. 2016;1(2):104-15.
16. Naressi WG, Naressi SCM, Orenha ES. Ergonomia e biossegurança em Odontologia. São Paulo: Artes Médicas, 2013. $128 \mathrm{p}$.

17. Garbin CAS, Saliba NA, Moimaz SAS, Santos KT. O papel das universidades na formação de profissionais na área de saúde. Rev ABENO. 2006;6(1):6-10.

18. Brasil. Conselho Nacional de Educação. Câmara de Educação Superior. Resolução CNE/CES 2, de 18 de junho de 2007. Dispõe sobre carga horária mínima e procedimentos relativos à integralização e duração dos cursos de graduação, bacharelados, na modalidade presencial. Diário Oficial da União, Brasília, Seção 1:6, 19 jun 2007. [Acesso em: 03/10/2016] Disponível em: http://portal.mec.gov.br/cne/arquivos/pdf/2 007/rces002_07.pdf

19. Musse JO, Boing AF, Martino FS, Silva RHA, Vaccarezza GF, Ramos DLP. O Ensino da bioética nos cursos de graduação em odontologia do estado de São Paulo. Arq Ciênc Saúde. 2007;14(1):13-6.

20. Pinheiro FMC, Nóbrega-Therrien SM, Almeida MEL, Almeida MI. A formação do cirurgião-dentista e a promoção de saúde no PSF. Rev Odontol UNESP. 2008;37(1):6977.

\section{Correspondência para:}

Suzely Adas Saliba Moimaz e-mail: sasaliba@foa.unesp.br Rua José Bonifácio, 1193 - Vila Mendonça 6015-050 Araçatuba/SP 